\title{
RELACIÓN ENTRE EL RENDIMIENTO DEL EXAMEN DE ADMISIÓN Y EL ACADÉMICO. TACNA, 2001- 2005
}

\author{
Responsable : : Lic. Gladys G. Benitez Palacios \\ Miembro : Mgr. Luz Marina Ticona Pajares
}

\section{RESUMEN}

El abandono y la prolongación de los estudios constituyen en la actualidad un importante problema en la Universidad. En el presente informe se presentan los resultados de un estudio transversal retrospectivo que tenia por objetivo establecer la relación entre el puntaje del examen de ingreso y el rendimiento académico de los ingresantes del 2001 a la Facultad de Ciencias de la Educación de la UNJBG. Se determinó que existe relación directa $(p<0,05)$ con una baja correlación $(r=0,34)$, esto, con escaso significado estadistico.

\begin{abstract}
Desertion and extension of studies are, at present, a great problem al the university. In this research we present some results of a retrospective-transversal study which goal was to stablish a relationship between the score of the Admission Exam and the Academic performance of the year 2001 from de Sciences of Education Faculty at the National University Jorge Basadre Grohmann. The research determined that there is a direct relationship $(p<0,05)$ with low correlation $(R=0,34)$, it means, lack of statistic meaning.
\end{abstract}

\section{INTRODUCCIÓN}

Es verdad admitida que la educación superior universitaria en el pais atraviesa por una serie de fenómenos que están provocando un desencuentro entre la universidad y las necesidades del pais. Las estadisticas universitarias estiman porcentajes de abandono de estudios sin finalizar de aproximadamente un 50\% (MEC: Catálogo de Indicadores del Sistema Universitario). Según Lidia Cabrera y otros (2005), el rendimiento académico de los estudiantes universitarios es un tema que preocupa por el gran costo social que supone un egresado, por el gasto añadido generado cuando un estudiante cursa estudios por más tiempo, o por el costo que no se rentabiliza cuando no finaliza, o por cuestiones de calidad, y rendimiento de cuentas social. Problema de similar naturaleza es la cantidad de años que el estudiante invierte en la obtención del título, muy por encima a los prescritos en el plan de estudios, cuando lo que se espera es que tanto la duración de los estudios como la titulación se aproximen a los plazos previstos para la carrera.

En la región Tacna, el año 2000 egresaron de la educación secundaria aproximadamente 6.800 estudiantes que en promedio tenían 17 años de edad; de estos postularon a la Universidad Nacional Jorge Basadre Grohomann 4 338, para cubrir 1585 vacantes (Oficina Admisión UNJBG). La Facultad de Ciencias de la Educación, para el mismo año, ofertó 255 vacantes en sus cinco especialidades. Si partimos del hecho que tanto el abandono como la deserción son problemas preocupantes, cabe preguntar: ¿Qué ha pasado con los ingresantes a la Facultad de Educación?, ¿cuál es su porcentaje de éxito?, ¿por cuánto tiempo se prolongan los estudios?, ¿existe relación entre los puntajes de ingreso y los promedios generales de egreso?

Aunque se han realizado pocas investigaciones formales a cerca de este tema, el conocimiento del comportamiento académico del estudiante toma cada vez mayor importancia para las técnicas y métodos de enseñanza moderna a fin de aplicarlas eficazmente. Según Adela Ferrante y Claudia Castro (1992), se entiende por rendimiento académico a los resultados o niveles alcanzados por el estudiante durante el proceso de enseñanza - aprendizaje y a este como la modificación del comportamiento a consecuencia de un proceso educativo.

El abandono de estudios y deserción estudiantil son términos que se utilizan para denominar una variedad de situaciones identificadas en el proceso educativo del estudiantado con un denominador común, detención o interrupción de estudios iniciados antes de finalizarlos. Esta categoría incluye: abandono involuntario (por incumplimiento administrativo o violación de reglamentos, traslado interno excepcional), dejar la carrera para iniciar otra en la misma institución (abandono por segunda opción), o para irse a otra universidad para completar estudios iniciados, renunciar a la formación universitaria para iniciar itinerarios formativos fuera de la universidad, o incorporarse al mundo laboral, interrumpir la formación con la intención de retomarla en el futuro, otras posibilidades.

La prolongación de estudios está más estrechamente vinculada con el concepto tradicional de fracaso académico. Se refiere a la diferencia del tiempo invertido con el teóricamente previsto para terminar la carrera. Este es uno más de los tantos indicadores que se han definido para identificar el fracaso académico (Latiesa, 1992).

Las estadisticas universitarias suelen identificar como "caso de abandono" al estudiante que ha iniciado estudios y, antes de finalizarlos, no se matricula en dos semestres consecutivos. Dentro de esta categoria, encontramos situaciones de estudiantes que deciden cambiar de carrera dentro de la misma universidad, o en otra universidad; estudiantes que hacen un paréntesis por razones múltiples. Existe la tendencia a aceptar que las tasas de abandono son un indicador de baja calidad: se entiende que la universidad no puso los medios necesarios para que los estudiantes alcanzaran la titulación. 
Al estudiar el abandono y la prolongación de estudios en la universidad, las investigaciones que siguen el modelo psicopedagógico, como las de González y col. (2005), analizaron factores personales, institucionales y sociales

Para algunos estudiantes, la adaptación a la vida universitaria constituye un reto y un compromiso personal que les lleva a esforzarse y a buscar la ayuda necesaria para alcanzar las metas que tienen planteadas. Sin embargo, otros muchos sucumben en el intento y se quedan a mitad de camino, pasando a engrosar la larga lista de los que fracasan. Por tanto, el alumnado con un perfil psicológico positivo para afrontar obstáculos obtiene una mayor adaptación y en consecuencia, una mayor persistencia. En este sentido, Kirton (2000) encontró que la percepción del ambiente universitario y la autoeficacia académica tenian una gran influencia en la persistencia académica por parte de los estudiantes universitarios de primer año, durante el primer semestre de curso. A través de análisis estadisticos de regresión jerárquica, Kirton (2000) identificó cinco factores como los más influyentes en las decisiones de persistencia del alumnado, en el logro de las metas académicas: la autoeficacia académica, los valores educativos, las percepciones del ambiente universitario, el apoyo universitario y el apego hacia los iguales.

Las variables institucionales, sobre todo las relacionadas con las caracteristicas de los estudios, los recursos académicos y el profesorado, han sido las más cuestionadas desde la opinión social en relación con el tema del abandono y prolongación de los estudios. Se considera que la politica universitaria se ha centrado en que acceda a la universidad el mayor número de usuarios posible, sin preocuparse en analizar los nuevos perfiles y necesidades del alumnado (preferencias profesionales, habilidades, capacidades, etc.), ni en poner los recursos necesarios para atender a todo el alumnado. Thomas (2002), en relación al rol que desempeña la institución en la decisión del alumnado de abandonar, atribuye una gran responsabilidad al profesorado, tanto a los métodos de enseñanza y modelos de evaluación utilizados como a las relaciones distantes que establece con sus alumnos, cuando éstos necesitan un trato más personalizado. Thomas considera que estas prácticas del profesorado, junto a la estructura institucional que tiende a reproducir una determinada cultura social, son factores que incitan al alumnado a abandonar. Cabrera y col. (2005) refieren que los resultados estadisticos mostraron que la titulación y el profesorado tenían un poder predictivo del abandono y prolongación de los estudios universitarios inferior a las variables del alumnado. Entre esas variables del contexto se encontró que ciertas características del profesorado (como "motivar al alumnado", "tener en cuenta sus opiniones sobre la asignatura", $y$ "dialogar con él sobre la marcha de las clases") se revelan como los aspectos más críticos de la enseñanza universitaria, que contribuyen a que el alumnado persista y finalice sus estudios

En cuanto a las variables del contexto referidas a las características de la titulación, Cabrera y col. encontrarón que el alumnado que abandona percibió que el nivel de exigencia de la titulación era muy alto, que tuvo poca información y ayuda durante los estudios, que no tuvo muy buenas relaciones con sus compañeros y que recibió escasa formación práctica. En este sentido, las expectativas y concepciones que tienen los estudiantes sobre la carrera, el nivel de exigencia y las relaciones entre los compañeros, son variables predictivas de éxito o fracaso académico, aspectos que tendrían que tenerse en cuenta en las acciones institucionales de información y orientación al alumnado, tanto antes como durante su estancia en la universidad.

En relación con el aspecto socioeconómico, los teóricos de la enseñanza universitaria refieren que la prolongación y el abandono de los estudios universitarios estaria influenciado, por un lado, por los cambios en la actividad económica productiva de las empresas (exige que los profesionales tengan un nuevo modelo de competencias, frente a lo cual han surgido un conjunto de agencias e instituciones educativas mucho más ágiles que la universidad que ofrecen los mismos servicios de una forma mucho más adaptada y accesible al puesto de trabajo); y, por otro, las bajas expectativas de empleo para los profesionales egresados .Abordar el estudio de este fenómeno exige ampliar el análisis desde el alumnado a la comunidad, pues con el abandono de los estudios son muchos los implicados como factores desencadenantes, y muchos los afectados. El primero es el alumnado, por el daño y sufrimiento psicológico que le produce esa experiencia de fracaso personal, que se extiende a su familia. La institución universitaria no queda exenta de frustración, pues los fracasos del alumnado remueven los cimientos institucionales, al no poder rendir cuentas socialmente desde los parámetros públicos de calidad. Finalmente, el tercer actor del fenómeno es el Estado, por el desequilibrio que supone para el sistema de educación superior, que afecta al conjunto social, por los enormes costos económicos que produce sin alcanzar los objetivos formativos poblacionales esperados.

\section{MATERIALY MÉTODOS}

El estudio se realizó con la población de ingresantes a la Facultad de Ciencias de la Educación de la UNJBG el año 2001, compuesta por 254 estudiantes distribuidos en cinco especialidades: Especialidad de Lengua, Literatura y Gestión Educativa (LEGE), 50 ingresantes (19,7\%); Especialidad de Idioma Extranjero, Traductor e Interprete (IETI), 51 ingresantes $(20,1 \%)$; Especialidad Ciencias Sociales y Promoción Sociocultural (SPRO), 51 ingresantes $(20,1 \%)$, Especialidad de Matemática, Computación e Informática (MACI), 51 ingresantes (20,1\%), Especialidad de Ciencias Naturales Tecnologia y Ambiente (NATA), 51 estudiantes $(20,1 \%)$.

La información fue recolectada del documento "Informe de Gestión Académica del Proceso de Admisión 2001 documento oficial de la Oficina de Admisión de la UNJBG, en el que se consigna información sobre las modalidades de ingreso y los puntajes obtenidos por la población de estudio. Los récord académicos de los estudiantes, documentos oficiales de la Sección de Registros Académicos de la Facultad de Ciencias de la Educación UNJBG, que contienen datos sobre la situación académica: notas por asignaturas, año en que se encuentra el estudiante, el número de veces que se matriculo por asignatura. El análisis estadístico se realizó en cuatro etapas: primero se determinó la modalidad de ingreso y los puntajes obtenidos por los ingresantes a las diferentes especialidades de la FACE; en segundo lugar, se estudió el porcentaje de estudiantes que culminaron su carrera con la titulación respectiva, los que aún se encuentran estudiando (prolongación de estudios) y los que 
habiendo ingresado ya no se encuentran matriculados (abandono de estudios); en tercer lugar, se establece el promedio ponderado de estudios académicos; $y$, finalmente, mediante el análisis de regresión lineal se estableció la relación entre los puntajes de admisión y los puntajes de rendimiento académico.

\section{RESULTADOS}

La Facultad de Ciencias de la Educación (FACE), el año 2001 cubre las vacantes ofertadas, por las diversas modalidades de admisión que ofrece la universidad. El 5,7; 12,2 y $45,12 \%$ ingresaron por la modalidad de examen de admisión Fase cero, Fase I y Fase II, respectivamente; en este caso los postulantes se someten a un examen de admisión y deben obtener un puntaje aprobatorio. El 21,96 $\%$ lo hace a través de la modalidad de CEPU, I, II, III; por esta modalidad ingresan los que siguen el ciclo de preparación intensiva programado por la UNJBG.

Un porcentaje pequeño $(10,16 \%)$ lo hace por la modalidad de admisión extraordinaria, en la que participan aquellos que tuvieron puntaje aprobatorio en el último examen de admisión (Fase II) y que inicialmente postularon a otra carrera profesional sin alcanzar vacante, otras modalidades $4,8 \%$. Al analizar los puntajes obtenidos en el examen de admisión, se observa que los puntajes oscilan entre 10,1 y 17,36 . El promedio mayor de ingreso es de 13,3 puntos en la especialidad de IETI, y el promedio menor $(12,6)$ en la especialidad de $\mathrm{MACl}$. Al compararlos con el resto de Facultades de la UNJBG, según las escalas propuesta por la Oficina de Admisión UNJBG, se encuentran en la escala de rendimiento regular. Por tanto, es probable que sea uno de los indicadores que influya en la prolongación y/o abandono de los estudios en la carrera iniciada.

Gráfico N $N^{\circ}$ 01: Facultad de Cs. de la Educación de la UNJBG-Tacna Ingresantes del 2001 por Año de Estudios y Según Promedios por Especialidad.

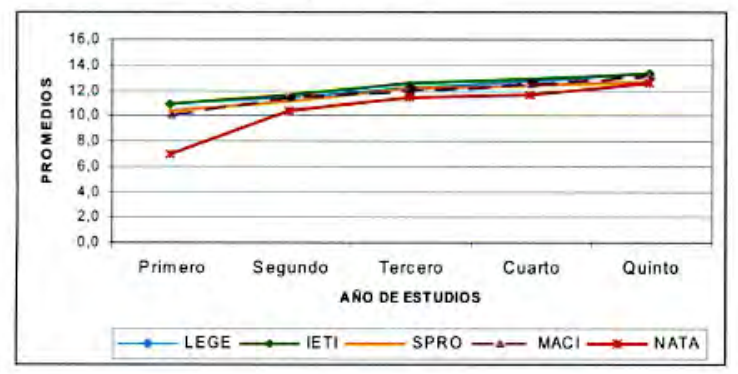

En el Gráfico $N^{\circ} 01$ se muestran los promedios del rendimiento académico de los estudiantes de las cinco especialidades de la FACE. Los mayores promedios, obtenidos durante los cinco años de su formación profesional, corresponden a los estudiantes de IETI (10,9 en primero, 11,6 en segundo, 12,6 en tercero, 12,9 en cuarto y 13,3 en quinto año); y los promedios más bajos, a los de NATA (7,0 en primero, 10,4 en segundo, 11,4 en tercero, 11,6 en cuarto, 12,6 en quinto). Se observa, en todos los casos, un ligero incremento en los promedios a medida que el estudiante avanza en su formación profesional.

Cuadro N ${ }^{\circ}$ 01: Facultad de Ciencias de la Educación de la UNJBG Ingresantes del 2001 por especialidades según situación académica.

\begin{tabular}{|c|c|r|r|r|r|r|}
\hline \multirow{2}{*}{ ESPECIALIDAD } & & \multicolumn{5}{|c|}{ SITUACIÓN ACADEMICA } \\
\cline { 2 - 7 } & & DESERCIÓN & $\begin{array}{r}\text { CONTINUAN } \\
\text { ESTUDIANDO }\end{array}$ & EGRESADOS & TITULADOS & TOTAL \\
\hline \multirow{2}{*}{ LEGE } & $\mathrm{N}^{\circ}$ & 15 & 14 & 4 & 17 & 50 \\
\cline { 2 - 7 } & $\%$ & 30,0 & 28,0 & 8,0 & 34,0 & 100 \\
\hline \multirow{2}{*}{ IETI } & $\mathrm{N}^{\circ}$ & 10 & 24 & & 17 & 51 \\
\cline { 2 - 7 } & $\%$ & 19,6 & 47,1 & 0,0 & 33,3 & 100 \\
\hline \multirow{2}{*}{ SPRO } & $\mathrm{N}^{\circ}$ & 19 & 15 & 3 & 14 & 51 \\
\cline { 2 - 7 } & $\%$ & 37,3 & 29,4 & 5,9 & 27,5 & 100,0 \\
\hline \multirow{2}{*}{ MACI } & $\mathrm{N}^{\circ}$ & 20 & 12 & 1 & 18 & 51 \\
\cline { 2 - 7 } & $\%$ & 39,2 & 23,5 & 2,0 & 35,3 & 100 \\
\hline \multirow{2}{*}{ NATA } & $\mathrm{N}^{\circ}$ & 39 & 8 & 1 & 3 & 51 \\
\cline { 2 - 7 } & $\%$ & 76,5 & 15,7 & 2,0 & 5,9 & 100 \\
\hline \multirow{2}{*}{ TOTAL } & $\mathrm{N}^{\circ}$ & 103 & 73 & 9 & 69 & 254 \\
\cline { 2 - 7 } & $\%$ & 40,6 & 28,7 & 3,5 & 27,2 & 100 \\
\hline
\end{tabular}

Fuente: Oficina de Registros Académicos FACE

En relación con la culminación (porcentaje de éxito), prolongación y abandono de estudios, los resultados muestran que durante el año académico 2006 obtuvieron su titulación el $27,2 \%$ de los estudiantes que ingresaron a la FACE el año 2001; estos serian los que lograron el éxito académico en el tiempo previsto en el plan de estudios (5 años), un porcentaje poco significativo $(3,5 \%)$ ha culminado estudios y está en condiciones de hacer los trámites para el proceso de bachillerato.

$\mathrm{Al}$ analizar los porcentajes por especialidades, se nota que las Especialidades de MACI (35,3\%) LEGE (34,0\%) y IETI $(33,3 \%)$ tienen porcentajes de titulados superiores al promedio de la Facultad. La Especialidad con menos titulados es la de NATA, (5,9\%).La prolongación de estudios, según De Miguel y Arias (1999), es un indicador de fracaso académico en la medida que se invierte un tiempo mayor que el teóricamente previsto para terminar los estudios. Este indicador es de $28,7 \%$ para la FACE. Hay diferencias importantes en cada una de las especialidades: IETI $(47,1 \%)$ muestra el porcentaje más alto de prolongación de estudios. Para la universidad, sin embargo, cualquier situación que impulse al alumnado a interrumpir sus estudios ha de ser valorada como fracaso, pues no se lograron los objetivos educativos del programa propuesto y enseñado.

Si se centra la atención en el fenómeno del abandono, esta cifra es muy alta, el $40,6 \%$ de los estudiantes que ingresaron en el año 2001 no registran matricula ni están en la lista de titulados o egresados de la Facultad. Esto significa que abandonaron la carrera, fenómeno crítico en la Especialidad de NATA, en la que el porcentaje de abandono se eleva a $76,5 \%$; mientras que en IETI es menor (19,7\%). En las Especialidades de LEGE y $\mathrm{MACl}$, el abandono de la carrera es de $30,0 \%$ y $39,2 \%$, respectivamente. Existe un consenso generalizado en aceptar que las tasas de abandono son un indicador de baja calidad, pues se entiende que la universidad y, en particular, la Facultad, no tomaron las medias necesarias para que los usuarios alcancen la titulación esperada. Estas medidas van asociadas, por una parte, con los requisitos previstos para el ingreso, de modo que el estudiante tome decisiones vocacionales acordes a sus perfiles personales (formación previa, aptitudes adecuadas a las materias que va a cursar, posibilidades económicas, adecuación vocacional de los estudios elegidos, motivación, etc.); y, por otra, durante el desarrollo de la carrera, con las estrategias formativas pertinentes a las necesidades de los destinatarios (adecuación de materias impartidas al número de créditos cursados; medios materiales y tecnológicos puestos al alcance del alumnado; apoyo psicopedagógico ofrecido por el centro, etc.). 
Gráfico N$^{\circ} 02$ : Facultad de Cs. de la Educación de la UNJBG -Tacna Ingresantes del 2001 por especialidades y según promedios en examen de admisión y rendimiento académico.

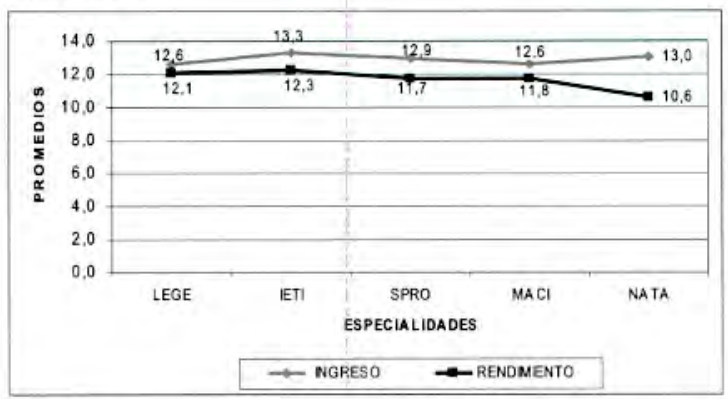

Al comparar los promedios del examen de admisión y el rendimiento académico de los ingresantes del 2001 a la FACE, se observa que los promedios de admisión por especialidades son mayores a los promedios del rendimiento académico. El mayor promedio, tanto en el examen de admisión como en el rendimiento académico, se observa en IETI $(13,3 ; 12,3)$ respectivamente, mientras que el promedio más bajo en admisión fue en la especialidad de $\operatorname{MACl}(12,6)$ y en el rendimiento académico fue la especialidad de NATA $(10,6)$.

Cuadro $N^{\circ}$ 02: Análisis de la relación entre los puntajes del examen de admisión y los promedios obtenidos durante su formación delos estudiantes ingresantes en el 2001 a la FACE.

\begin{tabular}{|c|c|c|c|c|c|c|c|}
\hline ESPECLALIDAO & $\begin{array}{l}\text { Fuente de } \\
\text { Variacion } \\
\end{array}$ & $\begin{array}{l}\text { Suma de } \\
\text { cuadrados }\end{array}$ & GI & \begin{tabular}{c|c} 
Media \\
cuadrática
\end{tabular} & $F$ & Sig. & $\mathbf{R}$ \\
\hline \multirow{3}{*}{ IETI } & Regresión & 3,33 & 1 & 3,33 & 0,31 & 0,58 & 0,08 \\
\hline & Residual & 516,19 & 48 & 10,75 & & & \\
\hline & Total & 519,52 & 49 & & & & \\
\hline \multirow{3}{*}{ LEGE } & Regresión & 47,27 & 1 & 47,27 & 10,79 & 0.00 & 0,44 \\
\hline & Residual & 192,72 & 44 & 4,38 & & & \\
\hline & Total & 240,00 & 45 & & & & \\
\hline \multirow{3}{*}{ MACl } & Regresión & 3,29 & 1 & 3,29 & 0,22 & 0,64 & 0,07 \\
\hline & Residual & 645,65 & 43 & 15,02 . & & & \\
\hline & Total & 648,94 & 44 & & & & \\
\hline \multirow{3}{*}{ NATA } & Regresión & 38,93 & 1 & 38,93 & 2.99 & 0,09 & 0.25 \\
\hline & Residual & 572.59 & 44 & 13,01 & & & \\
\hline & Total & 611,52 & 45 & & & & \\
\hline \multirow{3}{*}{ SPRO } & Regresión & 1.82 & 1 & 1,82 & 0.20 & 0,66 & 0,07 \\
\hline & Residual & 415,37 & 45 & $9,23$. & & & \\
\hline & Total & 417,19 & 46 & & & & \\
\hline \multirow{3}{*}{ FACULTAD (") } & Regresión & 10,94 & 1,00 & 10,94 & 9.82 & 0,00 & 0,34 \\
\hline & Residual & 84,69 & 76,00 & 1,11 & & & \\
\hline & Total & 95,63 & 77,00 & & 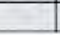 & & \\
\hline
\end{tabular}

* Se ha determinado la relación entre los puntajes de admisión y los de rendimiento académico de los estudiantes que culminaron sus estudios en los 5 años académicos estipulados en el plan de estudios.

Al relacionar estadisticamente el puntaje de ingreso y el rendimiento académico, puede visualizarse que existe relación directa $(p<0,05)$ con una baja correlación $(R=0,34)$, esto, con escaso significado estadistico, lo que implicaria que no necesariamente el estudiante que ingresa con alto puntaje ha tenido un rendimiento alto. Esto lleva a inferir que el proceso de admisión no cumple con el rol predictor que teóricamente debe tener.

\section{CONCLUSIONES}

Al sistematizar y analizar las variables ingreso, rendimiento académico y titulación prolongación y abandono de estudios, de los estudiantes que ingresaron a la Facultad de Ciencias de la Educación el año 2001, es posible afirmar que:

1. Los promedios obtenidos en el examen de admisión oscilan entre 12,6 y 13,3 puntos, y al comparar con el resto de Facultades, está dentro de aquellas cuyos promedios de ingreso son bajos. Es por tanto probable que éste sea uno de los indicadores que influya en la prolongación y/o abandono de los estudios en la carrera iniciada.

2. El rendimiento analizado a través de los puntajes promedios, se incrementa ligeramente a medida que el estudiante avanza en su formación profesional; sin embargo, estos indicadores se ubican en el límite medio de aprobación.

3. El porcentaje de los ingresantes que culmina su formación profesional en el tiempo señalado por el plan de estudios es de $27,2 \%$ en la Facultad. Las Especialidades de IETI, LEGE y MACI son las que tienen el mayor porcentaje de Licenciados (alrededor del $35 \%$ ), constituyendo el punto más critico la Especialidad de NATA, que solo llega a tener $5,9 \%$ de titulados.

4. El abandono de los estudios en la FACE es de $35 \%$, cifra mayor que la que se encuentra en otras universidades que reportan una tasa de abandono de $25 \%$. El mayor porcentaje se da en la Especialidad de NATA (70,6\%), seguido de SPRO $(35,3 \%)$, LEGE $(26,0 \%)$ y $\mathrm{MACl}$ $(27,5 \%)$.

5. El porcentaje de estudiantes que hacen la carrera en mayor tiempo que el establecido ( 5 años) difieren entre las especialidades, teniendo un mayor porcentaje la Especialidad de IETI (49,1\%), Las Especialidades de LEGE, SPRO y MACl, este porcentaje es alrededor del $40,6 \%$.

6. Al relacionar estadisticamente el puntaje obtenido en el examen de admisión y el puntaje de rendimiento académico se encontró relación directa $(P<0,05)$ con una baja correlación $(R=0,34)$, esto con escaso significado estadistico.

\section{BIBLIOGRAFIA}

Cano, E. (1998): La Evaluación de la calidad educativa, Madrid, La Muralla (2edición: 1999).

Casanova, $\mathbf{M}^{\mathrm{a}} \mathbf{A}(2004)$. Evaluación y Calidad de los centros educativos. Madrid, La Muralla S.A.

Casanova, Ma A (1992). La Evaluación Garantia de la Calidad para el Centro Educativo. Zaragoza, Edelvives.

Cabrera, L. Et Al (2006). Un estudio transversal retrospectivo sobre prolongación y abandono de estudios universitarios. RELIEVE, v. $12, n^{0} 1$.

et al (2006a) El problema del abandono de los estudios universitarios. RELIEVE, v. 12, $n^{\circ} 2$. http://www.uv.es/RELIEVE/v12n1/RELIEVEv12n1 1.ht m. Consultado en (28 de noviembre 2006).

Latiesa, M. (1992). La deserción universitaria. Madrid: CIS. 